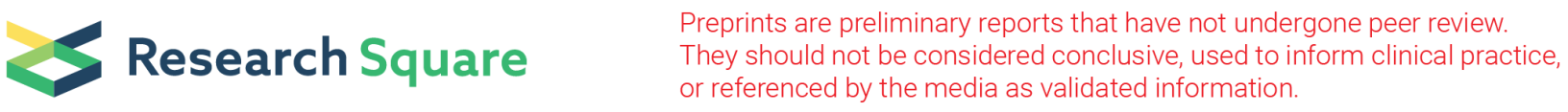

\title{
Leadership, Politics, and Communication: Epidemiology Workforce Roles and Challenges During Emergency Response; a Global Survey of Applied Epidemiologists, 2019-2020
}

Amy Elizabeth Parry ( $\nabla$ amy.parry@anu.edu.au )

Australian National University https://orcid.org/0000-0001-6641-7149

Martyn D Kirk

Australian National University

Samantha Colquhoun

Australian National University

David N Durrheim

University of Newcastle

Tambri Housen

University of Newcastle

\section{Research Article}

Keywords: Epidemiology, public health practice, health workforce, emergency, leadership, communication

Posted Date: January 19th, 2022

DOI: https://doi.org/10.21203/rs.3.rs-1239990/v1

License: (a) This work is licensed under a Creative Commons Attribution 4.0 International License. Read Full License 


\section{Abstract}

Background: Improving the epidemiological response to emergencies requires an understanding of who the responders are, their role and skills, and the challenges they face during responses.

Methods: We conducted a cross-sectional survey to learn more about epidemiologists who respond to public health emergencies. The online survey included open and closed-ended questions on challenges faced whilst responding, the roles of epidemiology responders, self-rating of skills, and support needed and received. We used purposive sampling to identify participants and a snowballing approach thereafter. We compared data by a number of characteristics, including national or international responder on their last response prior to the survey. We analysed the data using descriptive, content, and exploratory factor analysis.

Results: We received 166 responses from individuals with experience in emergency response. The most frequently reported challenge was navigating the political dynamics of a response, which was more common for international responders than national. National responders experienced fewer challenges related to culture, language, and communication. Epidemiology responders reported a lack of response role clarity, limited knowledge sharing, and communication issues during emergency response. Sixty-seven percent of participants reported they needed support to do their job well; males who requested support were statistically more likely to receive it than females who asked.

Conclusion: Our study identified that national responders have additional strengths such as better understanding the local political environment, language, and culture, which may in turn support identification of local needs and priorities. This research builds on emerging evidence on how to strengthen public health emergency response and provides a platform to begin a global conversation to address operational issues and the role of the international epidemiology responder.

\section{Background}

The global public health emergency response workforce deploy to control infectious disease outbreaks as well as respond to disasters to keep communities safe. To ensure that the public health response to events of international and national concern is timely and effective, we need to optimise workforce performance quality and impact.(1-3)

Health workforce development is a fundamental activity required to meet the obligations of the International Health Regulations (IHR 2005).(4) The Joint External Evaluation (JEE) is a tool aimed to identify how countries are tracking towards meeting these obligations. Findings from the JEE highlight that the public health workforce needs strengthening to effectively manage health security events.(5-8) In many countries, field epidemiology training programs (FETP) are implemented as a key activity to strengthen technical expertise within the existing workforce and to meet the target of 1 epidemiologist per 200,000 persons.(912)

There is limited evidence on best emergency response practice and there is a need for stronger scientific evidence and innovative research and evaluation methods.(13-16) The reasons for this are unclear but may relate to the lack of operational plan testing and poorly defined job roles. The lack of clarity on the role of the epidemiologist has been identified in previous research as a primary barrier to effective response. $(2,16,17)$

The COVID-19 pandemic has demonstrated the importance and value of the public health workforce, and the requirement for specialist epidemiology response skills and expertise. The Independent Panel's COVID-19 pandemic report and the 2021 World Health Assembly identified critical failures in responding to the COVID-19 crisis, including the under-resourced health workforce. $(18-20)$ It is clear that investing in and strengthening the public health emergency response workforce is an international priority. For well-targeted investment in workforce strengthening, we need to understand the challenges and barriers faced during public health emergency response.

International epidemiology emergency response work is most commonly short-term, which has led to a cycle of reaction during emergencies with limited or no documentation of the lessons learned, or modification of future response actions. After action reviews, or response evaluations, will go some way to addressing this, however they are an under-utilised tool.(21) For a stronger, 
better-managed epidemiology response, it is important to understand who the responders are, their role and skills, as well as how skills and knowledge are shared. In this study, we aimed to better understand the role of the epidemiologist and identify challenges faced during emergency response.

\section{Method}

We conducted a cross-sectional survey of emergency response epidemiologists. We have previously reported the methods of the survey elsewhere, and describe them here in brief.(2,22)

\section{Study population}

Participants of this survey were epidemiologists who had previously worked in an epidemiology role during an emergency response (Box 1).

Box 1

Terminology definitions provided to survey respondents

\section{Emergency response: A public health issue that requires external assistance.} This could mean a request for national and/or a request for international support. The crisis may be manmade (such as armed conflict), natural hazard (such as drought, flood) or the result of an infectious disease outbreak.

\section{Epidemiology role: The role performed by any person who participates in} surveillance, response, or disease control activities during an emergency.

\section{Sampling and recruitment}

Purposive sampling was initially used to identify participants, a snowballing method was then used to broaden participation. $(23,24)$

Recruitment consisted of multiple strategies. We produced a YouTube video to explain the purpose of the study; this video was produced in English and included optional multi-language subtitles. We disseminated the video via Twitter, Linkedln, and Facebook, and delivered eight reminders to participate via these platforms over three months. Our international partner, TEPHINET (Training Programs in Epidemiology and Public Health Interventions Network) supported recruitment through conducting a parallel social media campaign. Additionally, TEPHINET contacted FETP alumni and FETP country programs via email and through the TEPHINET website.

We advertised the survey at the 10th TEPHINET Global Scientific Conference, the Australian Communicable Diseases Control Conference 2019, and the European Scientific Conference on Applied Infectious Disease Epidemiology (ESCAIDE 2019). Survey respondents were requested to forward the survey link to other applied epidemiologists in their professional network.

\section{Data collection}

Survey questions were based on findings from key informant interviews conducted in 2019 and issues raised in the literature. $(16,25)$ Questions were reviewed by the key informant interviewees and pre-tested with 11 field epidemiologists with experience in emergency response. The survey was created and tested in English and then translated into French and back-translated to ensure accuracy. The survey was self-administered online by respondents via a REDCap (Research Electronic Data Capture) 
secure survey between October 2019 to February 2020. No incentives were provided for participation. The survey questionnaire is detailed elsewhere.(2)

In this analysis, we examined questionnaire data on [1] challenges faced whilst responding, [2] epidemiology responder roles, [3] self-rating of skills, and [4] support needed and received. Question format included checkbox items and Likert scales, each section included an optional open-ended question with free-text field, allowing participants to provide further comments. To contextualise answers, we asked respondents to reflect on their most recent emergency response experience. Due to the length of the survey, most questions were not compulsory; therefore, the denominator varies between questions.

\section{Ethics and Consent}

Participation in this study was voluntary and anonymous. We provided plain language study information in French and English. Each participant provided online consent before accessing the survey questions. During the survey, the participants could remove or change answers before survey submission. Ethics approval was provided by the Australian National University Human Research Ethics Committee (ID:2019-068).

\section{Data analysis}

We conducted descriptive analysis of survey data in Microsoft Excel and Stata15 (TX:StataCorp). We conducted content analysis on answers to open-ended questions; the text was open-coded and common categories were developed iteratively. $(26,27)$

In our analysis, we compared findings based on whether the participant's latest response was as an international or national responder (termed in this paper as 'responder type'). We also compared findings based on identified gender, epidemiology experience, epidemiology emergency response experience, and location. We grouped respondents by geographical areas according to the six World Health Organization (WHO) regions; Africa, Eastern Mediterranean, Europe, Americas, South-East Asia, and the Western Pacific.(28)

For questionnaire responses on roles and challenges, we performed an exploratory factor analysis to identify closely related items and distil the large dataset into fewer groupings. $(16,17)$ Eigenvalues and scree plots were reviewed to determine the number of groupings, orthogonal varimax rotation then supported the identification and interpretation of groupings of latent themes.(29) Factor loadings are the correlation between an item and a factor, a factor loading score from 0 to 1 indicates relationship between the item measured in that category.(29) Items were included in the factor if the loading was higher than 0.35.(29) The items found to contribute to a factor were then included in a scale where each item was equally weighted. For the scales identified through this factor analysis, Cronbach alpha (a) measured internal consistency between items was calculated to determine the scale reliability for future use. Typically, $a$ is considered reliable at $>0.7$, but moderate reliability can be identified at >0.6. $(30-32)$

To test for statistical difference, we used Pearson's chi-square for dichotomous data or two-sample Wilcoxon rank-sum (MannWhitney) test for data with multiple answer options. We considered results significant if they fell within the $95 \%$ confidence interval.

\section{Results}

\section{Participant characteristics}

We received 166 completed surveys, with representation from all WHO regions. There was a similar distribution of females and males among survey participants (Table 1). The majority of respondents were under 40 years old, with a median age of 39 (range: 23 to 77 years). A third of respondents reported less than five years of epidemiology experience and almost half had participated in three or fewer emergency response events in an epidemiology role (Table 1). Fifty-nine percent (96/162) reported their last response was a national response, while $41 \%$ (66/162) reported that it was an international response. 
Table 1

Demographics and characteristics of epidemiology emergency response survey participants $\left(n=166^{*}\right), 2019-2020$

\begin{tabular}{|c|c|c|}
\hline Category & Items & $n=166(100 \%)$ \\
\hline \multirow[t]{2}{*}{ Survey language } & English & $157(95 \%)$ \\
\hline & French & $9(5 \%)$ \\
\hline \multirow[t]{6}{*}{ Age (years) } & $<20$ & $0(0 \%)$ \\
\hline & $20-29$ & $10(6 \%)$ \\
\hline & $30-39$ & $78(47 \%)$ \\
\hline & $40-49$ & $47(28 \%)$ \\
\hline & $50-59$ & $25(15 \%)$ \\
\hline & $60+$ & $6(4 \%)$ \\
\hline \multirow[t]{3}{*}{ Identified gender } & Female & $85(51 \%)$ \\
\hline & Male & 79 (48\%) \\
\hline & Not reported & $2(1 \%)$ \\
\hline \multirow[t]{6}{*}{ Region (WHO region) } & African & $43(26 \%)$ \\
\hline & Eastern Mediterranean & $6(4 \%)$ \\
\hline & Europe & $20(12 \%)$ \\
\hline & Americas & $47(28 \%)$ \\
\hline & South-east Asia & $14(9 \%)$ \\
\hline & Western Pacific & $36(22 \%)$ \\
\hline \multirow{2}{*}{$\begin{array}{l}\text { Responder type } \\
(n=162)\end{array}$} & National & $96(59 \%)$ \\
\hline & International & $66(41 \%)$ \\
\hline \multirow[t]{2}{*}{ Training type } & FETP & $129(78 \%)$ \\
\hline & non-FETP & $37(22 \%)$ \\
\hline \multirow[t]{2}{*}{ Epidemiology experience $(n=164)$} & $<5$ years & $53(32 \%)$ \\
\hline & $5+$ years & $113(68 \%)$ \\
\hline \multirow[t]{2}{*}{ Any emergency response experience } & National $\leq 5$ events $(n=127)$ & $82(65 \%)$ \\
\hline & International $\leq 5$ events $(n=84)$ & $64(76 \%)$ \\
\hline \multirow[t]{2}{*}{ Epidemiology emergency response experience $(n=164)$} & $\leq 3$ events & $79(48 \%)$ \\
\hline & $4+$ events & $85(52 \%)$ \\
\hline \multirow[t]{6}{*}{ Emergency type experience ${ }^{\star \star}$} & Natural disaster & $70(42 \%)$ \\
\hline & Pandemic response & $50(30 \%)$ \\
\hline & Infectious disease outbreak response & $146(88 \%)$ \\
\hline & Conflict & $32(20 \%)$ \\
\hline & Refugee/displaced persons & $53(32 \%)$ \\
\hline & Nutrition emergency & $21(13 \%)$ \\
\hline
\end{tabular}




\begin{tabular}{|lll|}
\hline Category & Items & $\mathbf{n = 1 6 6 ( 1 0 0 \% )}$ \\
\hline Terms of Reference (ToR) & Other man-made disasters & $12(7 \%)$ \\
& Other & $9(5 \%)$ \\
\hline Contract length $(\mathrm{n}=163)$ & Had ToR $(\mathrm{n}=163)$ & $78(44 \%)$ \\
\cline { 2 - 3 } & ToR matched work $(\mathrm{n}=78)$ & $48(61 \%)$ \\
& Less than 1 month & $52(32 \%)$ \\
& $1-<2$ months & $37(23 \%)$ \\
& $2-<3$ months & $18(11 \%)$ \\
*denominator was 166 unless otherwise stated & $3-<6$ months & $20(12 \%)$ \\
**includes multiple answers per respondent & $6-<12$ months & $21(13 \%)$ \\
\hline
\end{tabular}

\section{Factor analysis}

We asked respondents to identify challenges they had experienced during their most recent emergency response (Table 2). Of the 18 listed challenges in the survey, exploratory factor analysis identified five factors with an eigenvalue over 1.(29) After scree plot review we reduced five contributing factors to four; 'communication', 'culture and relationships', 'technical skills', and 'political dynamics'.(33) One item, 'language', was not statistically related to other challenges therefore was analysed as a standalone item (Table 2). The Cronbach alpha for each of these scales was moderately reliable (>0.6) with the exception of 'political dynamics', indicating the series of questions could be useful in future analysis (Table 2).

The exploratory factor analysis of the 'epidemiology role' data condensed 37 items on epidemiology responder activities to nine factors with an eigenvalue of over 1.0.(29) After scree plot review we reduced the contributing factors to seven factors;(33) 'response guidance', 'data', 'investigation', 'surveillance', 'management', 'information', and 'cross-sectoral collaboration'(Table 3). These categories were not discrete, $5 \%(9 / 166)$ of respondents reported activities in a single category: $17 \%(29 / 166)$ reported activities across all seven categories (Table 3). The Cronbach alpha for each of these scales except for the role of 'information' and 'cross sectoral collaboration' were at least moderately reliable (>0.6) (Table 3). 
Table 2

Frequency of reported challenge groupings by epidemiology emergency response survey participants ( $\mathrm{n}=166), 2019-2020$

\begin{tabular}{|c|c|c|c|c|c|c|c|c|}
\hline Grouping & $\mathrm{n}=166(\%)$ & $\begin{array}{l}\text { Eigen } \\
\text { value }\end{array}$ & $\begin{array}{l}\text { Cronbach } \\
\text { a }\end{array}$ & $\begin{array}{l}\text { Itemised } \\
\text { challenge }\end{array}$ & $\begin{array}{l}\text { Factor } \\
\text { loading }\end{array}$ & $\mathrm{n}=166(\%)$ & $\begin{array}{l}\text { Responder } \\
\text { type* (p) }\end{array}$ & $\begin{array}{l}\text { Item-total } \\
\text { correlation }\end{array}$ \\
\hline \multirow[t]{7}{*}{$\begin{array}{l}\text { 1.Communication } \\
\text { ( } n=7 \text { challenges) }\end{array}$} & \multirow[t]{7}{*}{76 (46\%) } & 3.9 & \multirow[t]{7}{*}{0.74} & $\begin{array}{l}\text { 'Nobody told me } \\
\text { what had } \\
\text { already been } \\
\text { done' }\end{array}$ & 0.70 & 27 (16\%) & 0.09 & 0.68 \\
\hline & & & & $\begin{array}{l}\text { 'The team } \\
\text { leader did not } \\
\text { know how to } \\
\text { use me/my } \\
\text { skills' }\end{array}$ & 0.68 & 30 (18\%) & 0.049 & 0.65 \\
\hline & & & & $\begin{array}{l}\text { 'I did not } \\
\text { understand my } \\
\text { role' }\end{array}$ & 0.66 & 19 (11\%) & 0.26 & 0.70 \\
\hline & & & & $\begin{array}{l}\text { 'The priorities of } \\
\text { the response } \\
\text { were not } \\
\text { communicated } \\
\text { to me' }\end{array}$ & 0.63 & 22 (13\%) & 0.06 & 0.69 \\
\hline & & & & $\begin{array}{l}\text { 'Nobody told me } \\
\text { what to do' }\end{array}$ & 0.61 & 22 (13\%) & 0.34 & 0.68 \\
\hline & & & & $\begin{array}{l}\text { 'There were too } \\
\text { many other } \\
\text { epidemiologists' }\end{array}$ & 0.51 & $12(7 \%)$ & 0.50 & 0.50 \\
\hline & & & & $\begin{array}{l}\text { 'My deployment } \\
\text { period was too } \\
\text { short to be } \\
\text { effective' }\end{array}$ & 0.36 & 32 (19\%) & 0.69 & 0.48 \\
\hline \multirow[t]{4}{*}{$\begin{array}{l}\text { 2.Culture and } \\
\text { relationships } \\
\text { ( } n=4 \text { challenges) }\end{array}$} & \multirow[t]{4}{*}{$19(11 \%)$} & 2.1 & \multirow[t]{4}{*}{0.66} & $\begin{array}{l}\text { 'There was } \\
\text { nobody I could } \\
\text { ask cultural } \\
\text { questions to' }\end{array}$ & 0.82 & $2(1 \%)$ & 0.09 & 0.84 \\
\hline & & & & $\begin{array}{l}\text { 'I felt it was } \\
\text { difficult to } \\
\text { develop } \\
\text { relationships } \\
\text { with } \\
\text { international } \\
\text { colleagues' }\end{array}$ & 0.74 & $6(4 \%)$ & 0.003 & 0.69 \\
\hline & & & & $\begin{array}{l}\text { 'I did not } \\
\text { understand the } \\
\text { culture' }\end{array}$ & 0.60 & $9(5 \%)$ & 0.10 & 0.84 \\
\hline & & & & $\begin{array}{l}\text { 'I felt it was } \\
\text { difficult to } \\
\text { develop } \\
\text { relationships } \\
\text { with local } \\
\text { colleagues' }\end{array}$ & 0.60 & $10(6 \%)$ & 0.54 & 0.70 \\
\hline $\begin{array}{l}\text { 3.Technical skills } \\
\text { ( } n=3 \text { challenges) }\end{array}$ & 24 (14\%) & 1.6 & 0.67 & $\begin{array}{l}\text { 'I did not have } \\
\text { the right } \\
\text { technical skills } \\
\text { needed in the } \\
\text { field' }\end{array}$ & 0.75 & $12(7 \%)$ & 0.25 & 0.81 \\
\hline
\end{tabular}




\begin{tabular}{|c|c|c|c|c|c|c|c|c|}
\hline Grouping & $\mathrm{n}=166(\%)$ & $\begin{array}{l}\text { Eigen } \\
\text { value }\end{array}$ & $\begin{array}{l}\text { Cronbach } \\
\mathbf{a}\end{array}$ & $\begin{array}{l}\text { Itemised } \\
\text { challenge }\end{array}$ & $\begin{array}{l}\text { Factor } \\
\text { loading }\end{array}$ & $\mathrm{n}=166(\%)$ & $\begin{array}{l}\text { Responder } \\
\text { type * (p) }\end{array}$ & $\begin{array}{l}\text { Item-total } \\
\text { correlation }\end{array}$ \\
\hline & & & & $\begin{array}{l}\text { 'I found it } \\
\text { difficult to apply } \\
\text { my skills to the } \\
\text { required work' }\end{array}$ & 0.73 & $11(7 \%)$ & 0.74 & 0.78 \\
\hline & & & & $\begin{array}{l}\text { 'I did not have } \\
\text { the right } \\
\text { software skills' }\end{array}$ & 0.63 & 15 (9\%) & 0.09 & 0.74 \\
\hline \multirow[t]{3}{*}{$\begin{array}{l}\text { 4.Political } \\
\text { dynamics } \\
\text { ( } n=3 \text { challenges) }\end{array}$} & 88 (53\%) & 1.4 & 0.52 & $\begin{array}{l}\text { 'Political } \\
\text { dynamics were } \\
\text { challenging to } \\
\text { understand and } \\
\text { work within' }\end{array}$ & 0.73 & 58 (35\%) & 0.03 & 0.71 \\
\hline & & & & $\begin{array}{l}\text { 'Security issues } \\
\text { affected my } \\
\text { capacity to } \\
\text { work' }\end{array}$ & 0.64 & $36(22 \%)$ & 0.04 & 0.71 \\
\hline & & & & $\begin{array}{l}\text { 'Collaborating } \\
\text { with other } \\
\text { partners outside } \\
\text { my agency was } \\
\text { difficult' }\end{array}$ & 0.53 & 45 (27\%) & 0.552 & 0.72 \\
\hline $\begin{array}{l}\text { 5. Language } \\
\text { ( } n=1 \text { challenge) }\end{array}$ & $20(12 \%)$ & - & - & $\begin{array}{l}\text { 'My language } \\
\text { skills were } \\
\text { insufficient to } \\
\text { need' }\end{array}$ & 0.39 & 20 (12\%) & 0.001 & - \\
\hline
\end{tabular}


Table 3

Frequency of reported role groupings by epidemiology emergency response survey participants $(n=166) \star, 2019-2020$

\begin{tabular}{|c|c|c|c|c|c|c|c|c|}
\hline $\begin{array}{l}\text { Factor } \\
\text { grouping }\end{array}$ & $\begin{array}{l}\text { Factor } \\
\text { grouping } \\
n=166 \\
(\%)\end{array}$ & $\begin{array}{l}\text { Eigen } \\
\text { value }\end{array}$ & $\begin{array}{l}\text { Cronbach } \\
\text { a }\end{array}$ & $\begin{array}{l}\text { Responder } \\
\text { type }{ }^{\star \star}(p)\end{array}$ & Itemised activities & $\begin{array}{l}\text { Activities } \\
\text { reported } \\
n=166(\%)\end{array}$ & $\begin{array}{l}\text { Factor } \\
\text { loading }\end{array}$ & $\begin{array}{l}\text { Item-total } \\
\text { correlation }\end{array}$ \\
\hline \multirow{11}{*}{$\begin{array}{l}\text { Response } \\
\text { guidance } \\
\text { (n=11 } \\
\text { activities) }\end{array}$} & \multirow[t]{11}{*}{$\begin{array}{l}141 \\
(85 \%)\end{array}$} & 9.9 & \multirow[t]{11}{*}{0.84} & 0.15 & $\begin{array}{l}\text { Response activity } \\
\text { planning }\end{array}$ & $52(31 \%)$ & 0.72 & 0.74 \\
\hline & & & & & Sharing information & $87(52 \%)$ & 0.71 & 0.71 \\
\hline & & & & & Activity prioritisation & $47(28 \%)$ & 0.68 & 0.74 \\
\hline & & & & & $\begin{array}{l}\text { Communicating } \\
\text { findings }\end{array}$ & $86(52 \%)$ & 0.61 & 0.67 \\
\hline & & & & & Response evaluation & $43(26 \%)$ & 0.60 & 0.73 \\
\hline & & & & & Mentoring & $52(31 \%)$ & 0.57 & 0.55 \\
\hline & & & & & Report writing & $\begin{array}{l}100 \\
(60 \%)\end{array}$ & 0.52 & 0.64 \\
\hline & & & & & Needs assessment & $45(27 \%)$ & 0.47 & 0.56 \\
\hline & & & & & Risk assessment & $57(34 \%)$ & 0.45 & 0.55 \\
\hline & & & & & Research & $42(31 \%)$ & 0.41 & 0.50 \\
\hline & & & & & Mapping & $41(25 \%)$ & 0.41 & 0.49 \\
\hline \multirow{5}{*}{$\begin{array}{l}\text { Management } \\
\text { ( } n=5 \\
\text { activities) }\end{array}$} & \multirow[t]{5}{*}{$\begin{array}{l}107 \\
(65 \%)\end{array}$} & 1.6 & \multirow[t]{5}{*}{0.69} & 0.14 & $\begin{array}{l}\text { Team manager/ } \\
\text { supervisor }\end{array}$ & $50(30 \%)$ & 0.79 & 0.73 \\
\hline & & & & & Field coordinator & $50(30 \%)$ & 0.75 & 0.74 \\
\hline & & & & & $\begin{array}{l}\text { Epidemiology team } \\
\text { lead }\end{array}$ & $62(37 \%)$ & 0.46 & 0.60 \\
\hline & & & & & $\begin{array}{l}\text { Managing control } \\
\text { measures }\end{array}$ & $36(22 \%)$ & 0.44 & 0.70 \\
\hline & & & & & $\begin{array}{l}\text { Non-epidemiology } \\
\text { work }\end{array}$ & $47(28 \%)$ & 0.38 & 0.59 \\
\hline \multirow{2}{*}{$\begin{array}{l}\text { Cross- } \\
\text { sectoral } \\
\text { collaboration } \\
\text { (n=2 } \\
\text { activities) }\end{array}$} & \multirow[t]{2}{*}{$56(34 \%)$} & 1.2 & 0.58 & 0.004 & Source trace-back & $20(12 \%)$ & 0.67 & - \\
\hline & & & & & $\begin{array}{l}\text { Collaborating with } \\
\text { other sectors }\end{array}$ & $52(31 \%)$ & 0.63 & - \\
\hline \multirow{4}{*}{$\begin{array}{l}\text { Data } \\
\text { (n=4 } \\
\text { activities) }\end{array}$} & \multirow{4}{*}{$\begin{array}{l}119 \\
(72 \%)\end{array}$} & & \multirow[t]{4}{*}{0.89} & & Data cleaning & $79(48 \%)$ & 0.84 & 0.92 \\
\hline & & & & & Data management & $83(50 \%)$ & 0.83 & 0.88 \\
\hline & & & & & Data analysis & $\begin{array}{l}100 \\
(60 \%)\end{array}$ & 0.78 & 0.87 \\
\hline & & & & & Data entry & $68(41 \%)$ & 0.77 & 0.81 \\
\hline \multirow{4}{*}{$\begin{array}{l}\text { Investigation } \\
(\mathrm{n}=6 \\
\text { activities) }\end{array}$} & \multirow{4}{*}{$\begin{array}{l}113 \\
(68 \%)\end{array}$} & 2.2 & \multirow[t]{4}{*}{0.85} & 0.005 & Active case finding & $61(37 \%)$ & 0.81 & 0.81 \\
\hline & & & & & Contact tracing & $46(28 \%)$ & 0.78 & 0.75 \\
\hline & & & & & Interviews & $62(37 \%)$ & 0.76 & 0.81 \\
\hline & & & & & Case investigation & $72(43 \%)$ & 0.74 & 0.78 \\
\hline
\end{tabular}




\begin{tabular}{|c|c|c|c|c|c|c|c|c|}
\hline $\begin{array}{l}\text { Factor } \\
\text { grouping }\end{array}$ & $\begin{array}{l}\text { Factor } \\
\text { grouping } \\
\mathrm{n}=166 \\
(\%)\end{array}$ & $\begin{array}{l}\text { Eigen } \\
\text { value }\end{array}$ & $\begin{array}{l}\text { Cronbach } \\
\text { a }\end{array}$ & $\begin{array}{l}\text { Responder } \\
\text { type }{ }^{\star \star}(p)\end{array}$ & Itemised activities & $\begin{array}{l}\text { Activities } \\
\text { reported } \\
n=166(\%)\end{array}$ & $\begin{array}{l}\text { Factor } \\
\text { loading }\end{array}$ & $\begin{array}{l}\text { Item-total } \\
\text { correlation }\end{array}$ \\
\hline & & & & & Line listing & $60(36 \%)$ & 0.63 & 0.76 \\
\hline & & & & & Data collection & $82(49 \%)$ & 0.46 & 0.65 \\
\hline \multirow{5}{*}{$\begin{array}{l}\text { Surveillance } \\
\text { ( } \mathrm{n}=5 \\
\text { activities) }\end{array}$} & \multirow{5}{*}{$\begin{array}{l}118 \\
(71 \%)\end{array}$} & 2.0 & \multirow[t]{5}{*}{0.81} & 0.82 & Surveillance analysis & $84(51 \%)$ & 0.79 & 0.82 \\
\hline & & & & & $\begin{array}{l}\text { Surveillance } \\
\text { monitoring }\end{array}$ & $82(49 \%)$ & 0.74 & 0.73 \\
\hline & & & & & $\begin{array}{l}\text { Surveillance } \\
\text { evaluation }\end{array}$ & $54(33 \%)$ & 0.67 & 0.74 \\
\hline & & & & & Surveillance set up & $65(39 \%)$ & 0.53 & 0.75 \\
\hline & & & & & $\begin{array}{l}\text { Development of data } \\
\text { collection tools }\end{array}$ & $80(48 \%)$ & 0.48 & 0.74 \\
\hline \multirow{4}{*}{$\begin{array}{l}\text { Information } \\
\text { ( } n=4 \\
\text { activities) }\end{array}$} & \multirow[t]{4}{*}{$\begin{array}{l}112 \\
(67 \%)\end{array}$} & 1.4 & \multirow[t]{4}{*}{0.38} & 0.23 & $\begin{array}{l}\text { Transmission } \\
\text { analysis }\end{array}$ & $19(11 \%)$ & 0.63 & 0.56 \\
\hline & & & & & Response monitoring & $53(32 \%)$ & 0.50 & 0.67 \\
\hline & & & & & $\begin{array}{l}\text { Community } \\
\text { consultation }\end{array}$ & $29(17 \%)$ & -0.41 & 0.58 \\
\hline & & & & & Survey & $30(18 \%)$ & -0.54 & 0.54 \\
\hline $\begin{array}{l}\text { Other } \\
\text { (specified) }\end{array}$ & - & - & - & - & $\begin{array}{l}\text { Communications, } \\
\text { coordination, } \\
\text { infection prevention } \\
\text { \& control, collection \& } \\
\text { testing of specimens, } \\
\text { reviewing of } \\
\text { literature, treating } \\
\text { cases, vector control }\end{array}$ & - & - & - \\
\hline $\begin{array}{l}\text { * includes m } \\
\text { ** } \text { responder }\end{array}$ & $\begin{array}{l}\text { le answer } \\
\text { (internati }\end{array}$ & er resp & $\begin{array}{l}\text { ndent } \\
\text { ional respo }\end{array}$ & & & & & \\
\hline
\end{tabular}

\section{Communication}

Respondents highlighted the importance of good communication skills necessary for implementing interventions, community engagement, as well as for team functioning: "communication skills are key to successful public health interventions during a response. This goes for the community engagement side, but also within the team."

Respondents highlighted knowledge sharing as a challenge; commonly reporting a lack of recording of previous activities conducted by past deployees and poor handover. "Not enough existing handovers (in a written format, efficiently written, not just hidden in the mission report of another team member) led to less efficiency in the team." One respondent claimed, "existing staff acted as gatekeepers of knowledge, rather than centrally accessible information."

Almost 1:5 (32/166) of respondents reported that their deployment was too short for effective response (Table 2). The most common reported contract length was less than one month (32\%, 52/163), with 55\% (89/163) under two months (Table 1). One respondent reported, "you barely figure out what you are doing before you go home", and another stated that "it took time to develop relationships with colleagues until they were willing to allow me to have access to support the data management side of the work." The staff turnover also made it "difficult to know 'who-was-who' both among international and local teams". 


\section{Responder role}

Understanding the role of the epidemiology emergency responder was a common challenge identified. Eighteen percent of participants reported that 'The team leader did not know how to use me/my skills'(30/166), with significant difference noted between international and national responders (Table 2). The challenge of the team leader not knowing how to use the respondent was significantly different between respondents who had participated in three or fewer responses $(20 \%, 20 / 79)$ compared to those who reported four or more $(12 \%, 10 / 85)(p=0.02)$.

Individual misunderstanding of role was claimed to be from to a lack of clarity in job descriptions, less than $50 \%$ (78/162) of respondents reported they had Terms of Reference (ToR) for the last response they had undertaken (Table 2). Of respondents with a ToR, 61\% (48/78) of them stated they had a clear job description that matched the actual work they conducted (Table 1). "My ToR were vague and misleading." The lack of role clarity was commonly identified as a team or organisational issue, "the organisation I went out with was unsure what role they wanted to take within the larger epidemiological activities and as such my responsibilities were very unclear."

Respondents reported a common misunderstanding about the role difference between epidemiologists and medical professionals, with epidemiological work given to medical doctors. One respondent reported, "medical staff don't understand the role of the epidemiologist and sometimes attempt to do the work of the epidemiologist, which can lead to some really bad/messy data." In times where epidemiologists conducted epidemiological work, there was a lack of understanding by the team leaders on why the work (such as surveillance system development) was important, "[there were] Leaders with no public health or epi[demiology] background and [they] could not appreciate the importance of setting up surveillance."

A significant difference was detected between responder types and those working in a 'cross-sectoral collaboration'role $(p=0.004)$, with $23 \%(n=15 / 66)$ international responders reporting this role compared to $43 \%(n=41 / 96)$ of national responders (Table 3).

\section{Culture and relationships}

Few respondents identified items regarding 'culture and relationship' as a challenge (Table 2), and frequently self-rated their cross-cultural skills as strong (Figure 1). Contrary to these results, free text comments frequently discussed challenges in relationship formation, communication, and cross-cultural challenges.

Survey participants acknowledged the importance of understanding culture to enable effective investigation and control measure design. "My background is anthropology and I think it really helps when it comes to creating socially acceptable and impactful public health interventions". Some recommended cultural competence be included in briefings prior to deployment, whilst others discussed the importance of cultural mediators or community members in investigation teams; "training on specific cultural difference could be offered per country/region before/during a deployment" and "this is too often overlooked and many US county-level epidemiologists lack cultural competence."

It is easier to respond to health emergencies in your country of origin than in another country (can speak the language, familiarity with cultural, political issues). International response demands more personal skills (cultural sensitivity) and need for institutional support from WHO or sending agency along with clear ToR and command structure.

\section{Technical skills}

Participants reported few challenges regarding their technical skills (Table 2, Figure 1), however they recognised that technical knowledge alone was not enough to be effective during a response, "it's not just a matter of subject matter experts having the academic knowledge. They must have the additional skills to be productive and not a burden in an emergency."

Political dynamics 
The three individual challenges grouped in 'political dynamics' were the most listed challenges identified in the survey (Table 2). Navigating and understanding the political landscape was identified by $35 \%(58 / 166)$ of respondents, followed by the challenge of collaborating with external partners $27 \%(45 / 166)$. "You learn very quickly that interagency coordination with courteous communications is the key to success". International responders reported challenges with understanding the political environment of emergency response $(46 \%, 30 / 66)$, more than national responders $(29 \%, 28 / 96)(p=0.03)($ Table 2$)$.

Political issues identified from participant responses included: understanding the reasons behind decisions, power struggles, corruption, and politics within and between teams. Respondents reported difficulties collaborating within and between teams across the response, with trust identified as a key issue.

Fifty-nine percent (63/107) of those who reported undertaking management roles identified 'political dynamics'as a challenge compared to $42 \%(n=25 / 59)$ of non-management roles $(p=0.04)$. There was also a statistically significant difference between reporting 'political dynamics' as a challenge and those who worked on 'cross-sectoral collaboration' $(\mathrm{p}=0.006)$. Sixty-eight percent $(n=38 / 56)$ of those who reported a cross-sectoral collaboration role identified political dynamics as a challenge compared to $45 \%(n=50 / 110)$ of those who did not (Table 3$)$.

\section{Language}

When assessing self-confidence in skills, respondents reported the least confidence when working across languages (59\%, $83 / 140$ confident in this skill) (Figure 1). When comparing insufficient language ability to responder type, we found a statistical difference $(p=0.001)$ with $23 \%(15 / 66)$ of international responders stating their language skills were insufficient to need compared to $5 \%(5 / 96)$ of national responders (Table 2). Respondents reported that there was a need for interpreters when working across languages and that responders who spoke the local language should be prioritised.

\section{Other challenges}

Field experience was a common issue discussed by respondents. Respondents described a lack of support for new or inexperienced responders as well as the lack of emergency response entry-level positions. "Most emergency deployments require you to be able to function autonomously as an 'expert' epidemiologist, which is difficult for someone starting their career". Others reported that emergency response was not an appropriate place to train professionals. "Emergencies in remote locations or small countries often are not the right environment for support or professional development."

Respondents were concerned that post response evaluations were not occurring and that this limited the continuous improvement of responses. "Post response evaluation is seldom conducted. The idea like AAR [after action review] would be a good tool to regularly find out immediate issues and solve it to get prepared for the next event".

Additional challenges identified related to logistics and administration such as access to funding, cars, accommodation, internet, phone, and food. "Epidemiologists need to have some training related to resource management and basic understanding of finance and budgeting and costing of activities and plans", and "plain old admin [administration] support is necessary but not always provided."

\section{Support}

Reporting on their most recent emergency response, 67\% (103/153) of respondents stated they needed support to do their job well, 41\% (64/156) reported they needed technical epidemiological support and 46\% (72/156) non-technical support. Respondents commented on a need for the development of a pool of resources for use during emergency response and a contact list of experts available to answer questions.

Of those who stated they needed support, 77\% (77/103) reported they had received support. There was no statistical difference between identified gender on whether there was a need for support during the response, however, there was a difference between support received and gender $(\mathrm{p}=0.004)$, with more males reporting receiving support than females. There was a statistically significant difference in need of non-technical support between genders $(p=0.02)$ with $35 \%(30 / 85)$ of females reporting a need for non-technical support compared to $53 \%$ (42/79) of males.

Page $12 / 20$ 
Additionally, we identified a statistically significant difference between national and international responders and reported support received, $81 \%(52 / 64)$ and $64 \%(25 / 39)$, respectively $(p=0.03)$.

\section{Discussion}

The COVID-19 pandemic has exacerbated and bought to the fore the critical nature of a skilled, experienced, and well-trained public health workforce. Data for this study were collected prior to the COVID-19 pandemic, however are more poignant now than ever. Our research key findings can be consolidated into three principal themes: leadership, politics, and communication.

\section{Leadership}

\section{Epidemiology workforce role}

The need for local solutions to local problems is increasingly highlighted in the literature.(3,34-37) Our survey found that international responders reported more challenges than national responders in navigating the political environment, collaboration, security, and language. We must begin to think wider than individual roles and reconsider the broader use and role of both local and international responders during emergency response and how they can more effectively interact.

Many of the challenges identified in our study stem from the structure of emergency response. Short deployments of international responders with limited experience and little or no knowledge of the local language, culture, or politics, hampers effectiveness. Our findings showed that national responders have ancillary strengths, such as a better understanding of the political environment, language, and culture, which may better support the identification of local needs and priorities.(38-42)

The Global Preparedness Monitoring Board 2019 report indicated that emergency response systems need to better engage in community engagement during preparedness as well as response.(7) Local actors need to be at the centre of every response to ensure understanding of local context, history, cultural challenges. $(34,36)$ Increasingly, communities are demanding a leadership role within emergency response,(35) and countries are enforcing restrictions on international workers to ensure country coordination and management.(43) It has been noted that although international responders deploy with good intentions, they can obstruct the work on the ground.(43)

Just as the need for a rebalancing of power within the humanitarian aid sector between the Global North and South has been identified,(34) discussions also need to be had within the public health emergency response sector. The inequity of response between the Global South and Global North during the COVID-19 response further accentuates why a review of emergency response framework is needed.(44) We need to begin a global discussion ensuring adequate structures and frameworks for emergency response, and challenge the role of international responders to ensure leadership is centred at the local level to help address the complex challenges.(3,8) Emergency workforce response needs to move towards a national structure where international responders follow the lead of the national public health leadership and responders, and provide technical assistance to fill gaps and enhance capacity. $(3,34,38,45)$

This research identified the major role categories associated with the epidemiologist in emergency response. More work is needed to define roles by response type as well as to understand the minimum skills and experience needed to competently conduct each role, which in turn would inform the future field epidemiology and public health emergency response training.

Many of the challenges identified in this study are compounded by limited clarification or understanding of responder roles. Formalisation of required capacities and role of the medical and nursing professions during emergency response has been conducted, however, this has not yet been done for the epidemiology workforce.(46) Addressing clarity of roles for field epidemiologists will support effective recruitment, once required skills and experience are further refined. This would also lead to responders being more prepared for deployment, and therefore increasing their effectiveness.(17) 
There is a need to sensitise management and the broader emergency response community on the role of the epidemiology workforce so the skills they bring to a response are better understood and productively applied. Training of Public Health leaders and managers has begun in many countries, to broaden the understanding amongst leaders and managers of the value of the epidemiology workforce. We recommend this sensitisation of leaders be conducted within emergency response teams and their collaborators.

\section{Support}

Whilst short-term deployment of international responders with limited experience continues, response teams need support mechanisms that ensure the short deployments are effective. Enhanced identification of the national responder needs during response could support more effective targeted international deployments and remote support mechanisms.

In early 2021, the WHO Director-General stated that to strengthen health security, the global health emergency workforce needs investment and strengthening at all levels.(47) Although all levels do need strengthening, the primary focus should be to ensure local workforce support during emergencies to facilitate a reduction in long-term reliance on international deployments. Longerterm measures include addressing human resource issues and team structure to ensure collective competence during emergency responses. This includes training and upskilling of the new and current workforce, accreditation of the epidemiology profession, clarification of roles, defining minimum skills and experience needed for roles, and providing a support system to assist responders whilst working on an emergency.(16) Earlier research into the training gaps identified key areas for workforce support programs such as FETPs to focus on strengthening local workforces.(2) Other workforce support research has identified mentorship-type support helps to mitigate inexperience of the response workforce especially when navigating complex political environments. $(48,49)$

\section{Politics}

\section{Required skills}

The COVID-19 pandemic has clearly demonstrated that large public health events require responders with specialist skills and expertise to appropriately address the crisis. The pandemic response has also exposed the political nature of outbreaks and the critical role of politics in defining response direction and decision-making. $(3,18,19)$ Public health specialists and epidemiologists are critical to inform evidence-based decision making, however, the Independent Panel report decried political leadership that either failed to hear or act on this expertise to prevent SARS-CoV-2 transmission and guide control.(18) Our study has highlighted that epidemiology responders often do not understand the political dynamics of an outbreak, or find them difficult to navigate. Politics is a central component of outbreak management, and we need to better equip epidemiologists with political intelligence through future epidemiology training. $(37,48,50)$

\section{Gender}

Although our respondents were equally distributed across identified gender, we identified gender-based differences in who received needed support, with males receiving more support. In this increasingly female-dominated field, we need to do better to support women, especially when they request support. These findings are congruent with research and reports identifying gender biases in leadership and gender representation in global health.(3,8,51-53)

\section{Responder type}

When we compared the challenges and roles of national to international responders, we found that national responders were less likely to identify politics as a challenge and they were more likely to engage in cross-sectoral collaborative work. The importance of, and the need for, local based response is well documented in the literature.(39,54-59) Future outbreaks need to 
embrace this to ensure a one-health approach is taken to emergency response emergency, and collaboration rather than siloed work is essential.

\section{Communication}

Our research shows a broad range of communication challenges. In a recent study of epidemiology training needs, communication skill development was identified as in need of strengthening.(60) To improve the effectiveness of the epidemiology workforce during emergency response, communication strategies need to be developed. The COVID-19 pandemic has emphasised the need for clear communication, and prompt sharing of resources, information and knowledge. $(8,61,62)$ Improving communication skills of the epidemiology workforce would also support use of evidence to inform the response.(60) Defining and communicating the role of the epidemiologist to decision-makers and emergency responders would also help the broader response community to realise how the epidemiology workforce could be better integrated and utilised during a response. $(8,16)$

\section{Limitations}

Early consultations with emergency response organisations identified the absence of comprehensive emergency response workforce databases. This meant it was not possible to conduct representative sampling of this population. To lessen the impact of selection bias, we used multiple pathways to recruit participants. As this study included participants representing all WHO regions, we made the survey available in French and English to increase representation. There were varying timeframes between the most recent emergency response and the time of survey completion, potentially leading to different levels of recall. A time lag between responses and completion of the survey may have been advantageous as the individual had time to reflect on their role and the challenges they experienced. $(63,64)$

\section{Conclusion}

Leadership, politics, and communication skills need strengthening to ensure the public health workforce can effectively manage health security. Many of the challenges identified in this research stem from the current structure of global emergency response. We must begin a global conversation to reconsider the use and role of national and international responders during emergency response as the risk of another crisis similar or more severe than COVID-19 is plausible. The public health epidemiology workforce needs to be adequately and effectively prepared.

\section{Abbreviations}

AAR: after action review

COVID-19: Corona Virus Infectious Disease 2019

FETP: Field Epidemiology Training Programme

IHR: International Health Regulations

JEE: Joint External Evaluation

REDCap: (Research Electronic Data Capture)

TEPHINET: Training Programs in Epidemiology and Public Health Interventions Network

ToR: Terms of Reference

Page 15/20 


\section{Declarations \\ Ethics and consent:}

All activities were approved by the Science and Medical Delegated Ethics Review Committee at the Australian National University (protocol number 2019-068). All participants provided consent prior to accessing online survey.

\section{Author contributions:}

AP wrote the manuscript and is leading the study; AP, TH, DD, MK supported study design development, AP,TH, DD, MK, SC supported data analysis interpretation and revised the manuscript critically. All authors approved the final manuscript and agreed to be accountable for all aspects of the work.

\section{Competing interests:}

The authors declare that they have no competing interests.

\section{Funding:}

This research received no specific grant from any funding agency in the public, commercial or not-for-profit sectors. AP received Commonwealth and ANU science merit scholarships, along with funding from the Australian National Health Medical Research Council (NHMRC) Integrated Systems for Epidemic Response (APP1107393). MK was supported by an NHMRC Fellowship (APP1145997) and receives funding from the NHMRC for Integrated Systems for Epidemic Response. The funders did not have any role in the study design, data collection and analysis, decision to publish or preparation of the manuscript.

\section{Consent for publication:}

not applicable

\section{Availability of data and materials:}

All data relevant to this manuscript are included within the manuscript tables, figurers and/or reference links.

\section{Acknowledgements:}

Thank you to the participants of the survey for sharing your knowledge and experience. Thank you also to TEPHIconnect, FETP programme directors, and TEPHINET networks, for distributing the survey to members and for support with promotion. Thank you to Professor Alice Richardson and Associate Professor Dan Chateau for statistical advice.

\section{References}

1. Global strategy on human resources for health. Workforce 2030 [Internet]. [cited 2021 Jun 21]. Available from: https://www.who.int/publications-detail-redirect/9789241511131.

2. Parry A, Kirk M, Durrheim D, Olowokure B, Colquhoun S, Housen T. Shaping applied epidemiology workforce training to strengthen emergency response: a global survey of applied epidemiologists, 2019-2020. Hum Resour Health. 2021 Apr 
29;19(1):58.

3. Haldane V, Jung A-S, Foo CD, Bonk M, Jamieson M, Wu S, et al. Strengthening the basics: public health responses to prevent the next pandemic. BMJ. 2021 Nov;29:375:e067510.

4. WHO | International Health Regulations. (2005) [Internet]. WHO. [cited 2018 Apr 16]. Available from: http://www.who.int/ihr/publications/9789241580496/en/.

5. World Health Organization. Thematic Paper on the Status of Country Preparedness Capacities [Internet]. 2019 Sep [cited 2021 Jul 10]. Available from: https://apps.who.int/gpmb/assets/thematic_papers/tr-2.pdf.

6. Singh S, Bartos M, Abdalla S, Legido-Quigley H, Nordström A, Sirleaf EJ, et al. Resetting international systems for pandemic preparedness and response. BMJ. 2021 Nov 29;375:e067518.

7. Global Preparedness Monitoring Board. A world at risk: annual report on global preparedness for health emergencies [Internet]. Geneva: World Health Organization; 2019 [cited 2019 Sep 23]. Available from: https://apps.who.int/gpmb/assets/annual_report/GPMB_annualreport_2019.pdf.

8. Tan MMJ, Neill R, Haldane V, Jung A-S, Foo CD, Tan SM, et al. Assessing the role of qualitative factors in pandemic responses. BMJ. 2021 Nov 29;375:e067512.

9. WHO Regional Office for the Western Pacific. Asia Pacific strategy for emerging diseases and public health emergencies (APSED III): advancing implementation of the International Health Regulations. (2005) : working together towards health security [Internet]. Manila : WHO Regional Office for the Western Pacific; 2017 [cited 2020 Oct 21]. Available from: http://iris.wpro.who.int/handle/10665.1/13654.

10. O'Carroll PW, Kirk MD, Reddy C, Morgan OW, Baggett HC. The Global Field Epidemiology Roadmap: Enhancing Global Health Security by Accelerating the Development of Field Epidemiology Capacity Worldwide. Health Secur. 2021 Jun 1;19(3):34951.

11. O'Carroll P, Kirk M, Baggett K, Herrera D. The Global Field Epidemiology Roadmap [Internet]. 2018 [cited 2020 Sep 9]. Available from: https://www.tephinet.org/sites/tephinet/files/content/attachment/2018-1126/The\%20Global\%20Field\%20Epidemiology\%20Roadmap_11.26.18.FINAL_.pdf.

12. Williams SG, Fontaine RE, Turcios Ruiz RM, Walke H, ljaz K, Baggett HC. One Field Epidemiologist per 200,000 Population: Lessons Learned from Implementing a Global Public Health Workforce Target. Health Secur. 2020 Jan 1;18(S1):S-113-S118.

13. Blanchet K, Ramesh A, Frison S, Warren E, Hossain M, Smith J, et al. Evidence on public health interventions in humanitarian crises. The Lancet. 2017 Nov 18;390(10109):2287-96.

14. Blanchet K. Bayard Roberts. An evidence review of research on health interventions in humanitarian crises. London School of Hygiene and Tropical Medicine. Harvard School of Public Health, Overseas Development Institute; 2015 Oct.

15. Roy N, Thakkar P, Shah H. Developing-World Disaster Research: Present Evidence and Future Priorities. Disaster Med Public Health Prep. 2011 Jun;5(02):112-6.

16. Parry AE, Kirk MD, Durrheim DN, Olowokure B, Colquhoun S, Housen T. Emergency response and the need for collective competence in epidemiological teams. Bull World Health Organ. 2021 Mar;99(5)(2):351-8.

17. Hunt MR. Moral experience of Canadian healthcare professionals in humanitarian work. Prehospital Disaster Med. 2009 Dec;24(6):518-24.

18. Sirleaf EJ, Clark H. Report of the Independent Panel for Pandemic Preparedness and Response: making COVID-19 the last pandemic. The Lancet [Internet]. 2021 May 12 [cited 2021 May 19];0(0). Available from: https://www.thelancet.com/journals/lancet/article/PIIS0140-6736(21)01095-3/abstract.

19. The Independent Panel for Pandemic Preparedness and Response. COVID-19: make it the last pandemic [Internet]. [cited 2021 May 21]. Available from: https://theindependentpanel.org/.

20. Update from the Seventy-fourth World Health Assembly. - 28 May 2021 [Internet]. [cited 2021 Jun 21]. Available from: https://www.who.int/news/item/28-05-2021-update-from-the-seventy-fourth-world-health-assembly-28-may-2021.

21. Dalton CB, Kirk MD, Durrheim DN. Using after-action reviews of outbreaks to enhance public health responses: lessons for COVID-19. Med J Aust [Internet]. 2021 Sep 27 [cited 2021 Dec 9];Online first. Available from:

Page $17 / 20$ 
https://www.mja.com.au/journal/2021/using-after-action-reviews-outbreaks-enhance-public-health-responses-lessonscovid-19.

22. Parry AE, Kirk MD, Durrheim DN, Olowokure B, Housen T. Study protocol: building an evidence base for epidemiology emergency response, a mixed-methods study. BMJ Open. 2020 Jun 1;10(6):e037326.

23. Deborah K. Padget. Qualitative and Mixed Methods in Public Health. SAGE Publications; 2012.

24. Teddlie C, Yu F. Mixed Methods Sampling: A Typology With Examples. J Mix Methods Res. 2007 Jan;1(1):77-100.

25. Parry AE, Kirk MD, Colquhoun SM, Housen T. Enhancing the effectiveness of the epidemiology workforce responding to Covid-19. BMJ [Internet]. 2020 Mar 19 [cited 2020 Jul 21];368(m1075). Available from: https://www.bmj.com/content/368/bmj.m1075/rr-0.

26. Liamputtong P. Qualitative Research Methods. South Melbourne, Vic. Oxford University Press; 2009.

27. Braun V, Clarke V. Using thematic analysis in psychology. Qual Res Psychol. 2006 Jan 1;3(2):77-101.

28. WHO Regional Offices [Internet]. [cited 2020 Sep 30]. Available from: https://www.who.int/about/who-we-are/regionaloffices.

29. Tabachnick B, Fidell L. Using Multivariate Statistics: Pearson New International Edition PDF EBook [Internet]. Harlow, UNITED KINGDOM: Pearson Education, Limited; 2013 [cited 2021 Sep 15]. Available from:

http://ebookcentral.proquest.com/lib/anu/detail.action?doclD=5175291.

30. Taber KS. The Use of Cronbach's Alpha When Developing and Reporting Research Instruments in Science Education. Res Sci Educ. 2018 Dec;48(6)(1):1273-96.

31. Bland JM, Altman DG. Statistics notes: Cronbach's alpha. BMJ. 1997 Feb 22;314(7080):572.

32. Tavakol M, Dennick R. Making sense of Cronbach's alpha. Int J Med Educ. 2011 Jun 27;2:53-5.

33. Factor Analysis. A Short Introduction, Part 4-How many factors should I find? [Internet]. The Analysis Factor. 2012 [cited $2021 \mathrm{Jul}$ 27]. Available from: https://www.theanalysisfactor.com/factor-analysis-how-many-factors/.

34. Decolonising Humanitarianism or Humanitarian Aid? [Internet]. Speaking of Medicine and Health. 2021 [cited 2021 Jul 14]. Available from: https://speakingofmedicine.plos.org/2021/07/13/decolonising-humanitarianism-or-humanitarian-aid/.

35. Alice Chatelet; Meg Sattler. Coordinating a revolution: the critical role of response leadership in improving collective community engagement [Internet]. United Kingdom: Humanitarian Practice Network and Overseas Development Institute; 2019 Feb [cited 2019 Mar 22]. (Humanitarian Exchange). Report No.: 74. Available from: https://odihpn.org/wpcontent/uploads/2019/02/HE-74-web.pdf.

36. de Geoffroy V, Grunewald F. More than the money: Localisation in Practice [Internet]. Trocaire and Groupe URD; 2017 Jul [cited 2018 May 24]. Available from: https://charter4change.files.wordpress.com/2017/09/more-than-the-money-fullreport.pdf.

37. Haldane V, Jung A-S, Neill R, Singh S, Wu S, Jamieson M, et al. From response to transformation: how countries can strengthen national pandemic preparedness and response systems. BMJ. 2021 Nov 29;375:e067507.

38. Abimbola S, Asthana S, Montenegro C, Guinto RR, Jumbam DT, Louskieter L, et al. Addressing power asymmetries in global health: Imperatives in the wake of the COVID-19 pandemic. PLOS Med. 2021 Apr;22(4):e1003604. 18(.

39. Ehiri JE, Gunn JKL, Center KE, Li Y, Rouhani M, Ezeanolue EE. Training and deployment of lay refugee/internally displaced persons to provide basic health services in camps: a systematic review. Glob Health Action [Internet]. 2014 Oct 1 [cited 2018 May 30];7. Available from: https://www.ncbi.nlm.nih.gov/pmc/articles/PMC4185139/.

40. Chiu C-Y, Lonner WJ, Matsumoto D, Ward C. Cross-Cultural Competence. J Cross-Cult Psychol. 2013;44(6):843-8.

41. Hunt MR. Establishing moral bearings: ethics and expatriate health care professionals in humanitarian work. Disasters. 35(3):606-22.

42. Johnson. How language problems bedevil the response to crises. The Economist [Internet]. 2018 Nov 15 [cited 2018 Nov 19]; Available from: https://www.economist.com/books-and-arts/2018/11/17/how-language-problems-bedevil-the-response-tocrises?fsrc=scn\%2Ffb\%2Fte\%2Fbl\%2Fed\%2Fhowlanguageproblemsbedeviltheresponsetocrisesjohnson. 
43. Why Indonesia's rules on foreign tsunami relief are rattling the aid sector [Internet]. IRIN. 2018 [cited 2018 Oct 22]. Available from: https://www.irinnews.org/analysis/2018/10/16/why-indonesia-s-rules-foreign-tsunami-relief-are-rattling-aid-sector.

44. Asiimwe S, Nakku-Joloba E, Semeere A. Isolated in Uganda: How Covid-19 evacuations highlighted unfairness of global health partnerships. StatNews [Internet]. 2021 Nov 12 [cited 2021 Nov 15]; Available from: https://www.statnews.com/2021/11/12/isolated-in-uganda-covid-19-evacuations-highlights-unfairness-global-healthpartnerships/.

45. International Federation of Red Cross and Red Crescent Societies. FROM WORDS TO ACTION: Towards a communitycentred approach to preparedness and response in health emergencies [Internet]. 2019 Sep [cited 2021 Jul 10]. Available from: https://apps.who.int/gpmb/assets/thematic_papers/tr-5.pdf.

46. Foreign Medical Team Working Group under the Global Health Cluster. Norton I, Schreeb J von, Aitken P, Herard P, Lajolo C. Classification and minimum standards for foreign medical teams in sudden onset disasters [Internet]. World Health Organization;; [cited 2018 Jul 23]. Available from:

http://www.who.int/hac/global_health_cluster/fmt_guidelines_september2013.pdf?ua=1.

47. World Health Organization Director General. WHO's work in health emergencies, Strengthening WHO's global emergency preparedness and response. 2021 Jan p. 5. (148th session). Report No.: EB148/18.

48. Parry A, Colquhoun S, Brownbill A, Lynch B, Housen T. Navigating Uncertainty: Evaluation of a COVID-19 Surge Workforce Support Program, Australia 2020-2021. Glob Biosecurity [Internet]. 2021 Nov 24 [cited 2021 Dec 9];3(1). Available from: http://jglobalbiosecurity.com/articles/10.31646/gbio.124/.

49. Parry AE, Colquhoun SM, Field E, Kirk M, Durrheim D, Housen T. How can we better support the public health emergency response workforce during crises? Public health emergency response workforce support. West Pac Surveill Response. 2021 Nov 23;12(4):3-3.

50. Madhu Pai MD. PhD. The famous "epidemiological triad or triangle" needs an update (during Covid-19) https://t.co/t4X59csBRq [Internet]. @paimadhu. 2021 [cited 2021 Jul 26]. Available from: https://twitter.com/paimadhu/status/1396459426832429057.

51. Dhatt R, Theobald S, Buzuzi S, Ros B, Vong S, Muraya K, et al. The role of women's leadership and gender equity in leadership and health system strengthening. Glob Health Epidemiol Genomics [Internet]. 2017 ed [cited 2020 Oct 27];2. Available from: https://www.cambridge.org/core/journals/global-health-epidemiology-and-genomics/article/role-ofwomens-leadership-and-gender-equity-in-leadership-and-health-systemstrengthening/A6AEB63AFE17295E0EF9E40741A2EC5B.

52. Sumegha Asthana SE, Davies R, Dhatt A, Keeling A, Lal A, Phelan, et al. Strengthen Gender Mainstreaming in WHOs Pandemic Preparedness and Response [Internet]. 2020 Oct [cited 2020 Oct 29]. Available from: https://www.genderandcovid-19.org/wp-content/uploads/2020/10/Strengthen-gender-mainstreaming-in-WHOs-pandemicpreparedness-and-response.pdf.

53. Smith J. Overcoming the 'tyranny of the urgent': integrating gender into disease outbreak preparedness and response. Gend Dev. 2019 May 4;27(2):355-69.

54. Yozwiak NL, Happi CT, Grant DS, Schieffelin JS, Garry RF, Sabeti PC, et al. Roots, Not Parachutes: Research Collaborations Combat Outbreaks. Cell. 2016 Jun 30;166(1):5-8.

55. Currie J, Grenfell B, Farrar J. Beyond Ebola. Science. 2016 Feb 19;351(6275):815-6.

56. Bower H. Putting the capacity into capacity building in South Sudan. The Lancet. 2000 Aug;19(9230):661. 356(.

57. Buseh AG, Stevens PE, Bromberg M, Kelber ST. The Ebola epidemic in West Africa: Challenges, opportunities, and policy priority areas. Nurs Outlook. 2015 Jan;63(1):30-40.

58. UNHCR - Rohingya. refugee volunteers trained to help others in distress [Internet]. [cited 2018 May 30]. Available from: http://www.unhcr.org/news/latest/2018/5/5b066a544/rohingya-refugee-volunteers-trained-help-others-distress.html.

59. André AM, Lopez A, Perkins S, Lambert S, Chace L, Noudeke N, et al. Frontline Field Epidemiology Training Programs as a Strategy to Improve Disease Surveillance and Response. Emerg Infect Dis. 2017 Dec;23(Suppl 1):166-73. 
60. Parry AE, Kirk MD, Durrheim DN, Olowokure B, Colquhoun SM, Housen T. Shaping applied epidemiology workforce training to strengthen emergency response: a global survey of applied epidemiologists, 2019-2020. Hum Resour Health. 2021

Dec;19(1):58.

61. Hyland-Wood B, Gardner J, Leask J, Ecker UKH. Toward effective government communication strategies in the era of COVID19. Humanit Soc Sci Commun. 2021 Dec;8(1):30.

62. Lencucha R, Bandara S. Trust, risk, and the challenge of information sharing during a health emergency. Glob Health. 2021 Feb 18;17(1):21.

63. Bernard AW, Gorgas D, Greenberger S, Jacques A, Khandelwal S. The Use of Reflection in Emergency Medicine Education. Acad Emerg Med. 2012;19(8):978-82.

64. World Health Organization. Guidance for after action review (AAR) [Internet]. Geneva, Switzerland: World Health Organization; 2019 [cited 2019 Oct 8]. Report No.: WHO/WHE/CPI/2019.4. Available from:

https://apps.who.int/iris/bitstream/handle/10665/311537/WHO-WHE-CPI-2019.4-eng.pdf?sequence=1.

\section{Figures}

I am confident in effectively communicating with local communities about their

local beliefs and practices that may be barriers to response efforts

I am confident when working with communities and other local stakeholders to develop culturally sensitive approaches to responding to the emergency

I am confident when prioritising needs during an emergency response

I am confident in recognising when a cross-cultural misunderstanding has occurred

I am confident when I have to quickly adjust to changes I have no control over

I am confident in communicating with community members to understand local perspectives related to public health issues

I am confident when I have multiple important tasks competing for my time

I am confident when working with colleagues when we do not speak the same language

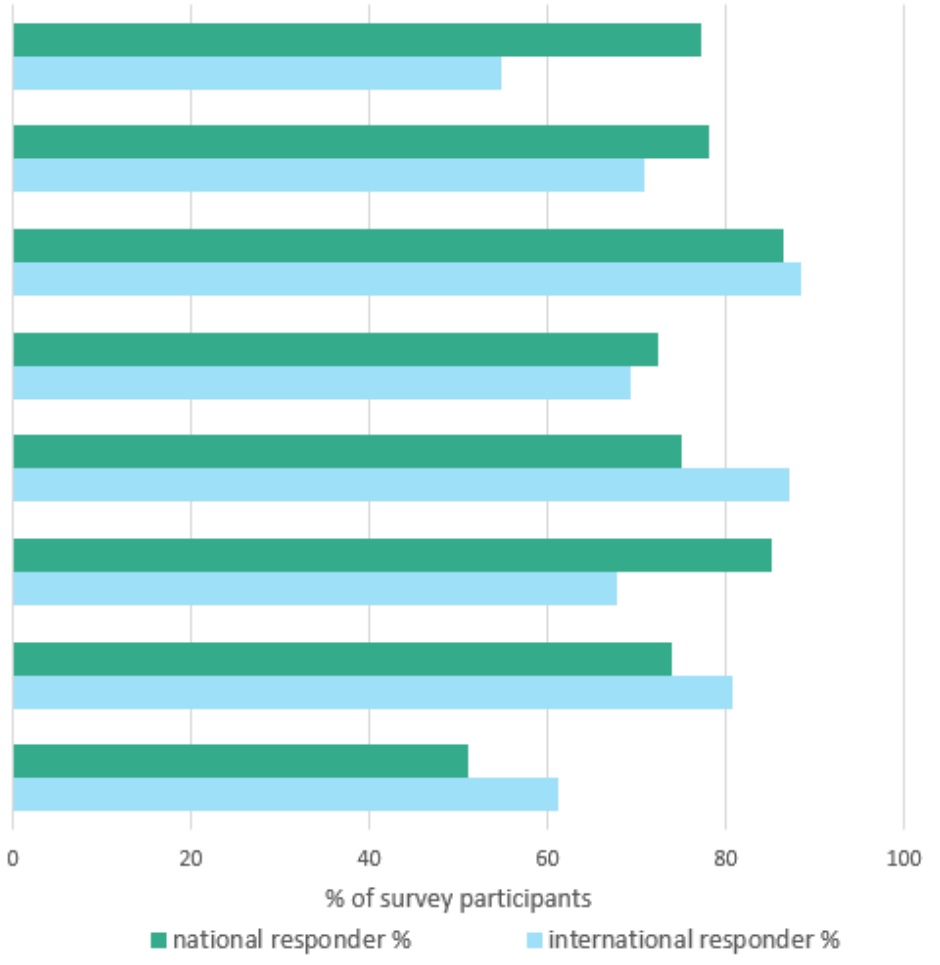

Figure 1

Comparison of international and national epidemiology emergency responders self-reported confidence in skills, 2019-2020 